\title{
FinFET SRAM Cell Optimization Considering Temporal Variability Due to NBTI/PBTI, Surface Orientation and Various Gate Dielectrics
}

\author{
Vita Pi-Ho Hu, Student Member, IEEE, Ming-Long Fan, Student Member, IEEE, Chien-Yu Hsieh, \\ Pin Su, Member, IEEE, and Ching-Te Chuang, Fellow, IEEE
}

\begin{abstract}
This paper analyzes the impacts of intrinsic process variations and negative bias temperature instability (NBTI)/positive BTI (PBTI)-induced time-dependent variations on the stability/variability of 6T FinFET static random access memory (SRAM) cells with various surface orientations and gate dielectrics. Due to quantum confinement, (110)-oriented pulldown n-channel FETs with fin line-edge roughness (LER) show larger Vread,0 and Vtrip variations, thus degrading READ static noise margin (RSNM) and its variability. Pull-up p-channel FETs with fin LER that are (100)-oriented show larger Vwrite,0 and Vtrip variations, hence degrade the variability of WRITE SNM. The combined effects of intrinsic process variations and NBTI/PBTI-induced statistical variations have been examined to optimize the FinFET SRAM cells. Worst-case stress scenario for SNM stability/variability is analyzed. With the presence of both NBTI and PBTI in high- $k$ metal-gate FinFET SRAM, the RSNM suffers significant degradation as Vread,0 increases, whereas Vtrip simultaneously decreases. Variability comparisons for FinFET SRAM cells with different gate stacks $\left(\mathrm{SiO}_{2}\right.$ and $\left.\mathrm{SiO}_{2} / \mathrm{HfO}_{2}\right)$ are also examined. Our paper indicates that the consideration of NBTI/PBTI-induced temporal variation changes the optimal choice of FinFET SRAM cell surface orientations in terms of the $\mu / \sigma$ ratio in RSNM.
\end{abstract}

Index Terms-FinFET, negative bias temperature instability (NBTI), positive bias temperature instability (PBTI), static random access memory (SRAM), surface orientation, variability.

\section{INTRODUCTION}

M ULTIGATE FinFETs are promising device candidates for post-22-nm complementary metal-oxidesemiconductor (MOS) technology generations due to their superior short channel effects, better subthreshold slope, and reduced random dopant fluctuation. The sidewall surface (conducting channel) orientation of FinFET devices can be easily changed by rotating the layout of the devices to improve the

Manuscript received August 5, 2010; revised October 25, 2010 and November 29, 2010; accepted December 3, 2010. Date of publication January 10, 2011; date of current version February 24, 2011. This work was supported in part by the National Science Council of Taiwan under Contract NSC 99-2221-E-009-174, in part by the Ministry of Education in Taiwan under the Aiming for the Top University Program, and in part by the Ministry of Economic Affairs in Taiwan under Contract 98-EC-17-A-01-S1-124. The authors are grateful to National Center for High-Performance Computing in Taiwan for computational facilities and software. The review of this paper was arranged by Editor J. S. Suehle.

The authors are with the Department of Electronics Engineering and the Institute of Electronics, National Chiao Tung University, Hsinchu 30050, Taiwan (e-mail: vitabee.ee93g@nctu.edu.tw; pinsu@faculty.nctu.edu.tw).

Color versions of one or more of the figures in this paper are available online at http://ieeexplore.ieee.org.

Digital Object Identifier 10.1109/TED.2010.2099661 carrier mobility, and thus circuit performance [1]-[3]. The fluctuation of the fin width due to line-edge roughness (fin LER) is widely recognized as a major source of variability for FinFET devices [4]. As the fin width scales down, the quantum-mechanical effect becomes more significant. However, the different surface orientation, with different quantization effective mass and quantum confinement, may result in distinctly different variability [22], [25].

In addition to time-zero intrinsic process variability, negative bias temperature instabilities [NBTI; for p-channel FET (PFET)] and positive BTI [PBTI; for n-channel FET (NFET)] have become major long-term reliability concerns as they weaken MOSFETs over time, thus resulting in temporal degradation in the stability and variability of the static random access memory (SRAM) cells [5]-[9]. The (110)-oriented Si surface has more dangling bonds before passivation and is therefore expected to have more bonded hydrogen at the interface in comparison with (100)-oriented Si surface. As such, the NBTI/PBTI degradation is more significant in (110)-oriented device than in (100)-oriented one [10], [11]. FinFET devices with different surface orientations exhibit distinct threshold voltage variations resulting from the intrinsic process variations and NBTI/PBTIinduced temporal variations. Fig. 1(a)-(c) illustrate the layouts of 6T FinFET SRAM cells with various combination of (110) and (100) surface (conducting channel) orientations by rotating the FinFET devices. The layouts are based on scaled ground rules from 32-nm node according to the International Technology Roadmap for Semiconductors projection.

In this paper, for the first time, the combined effects of time-zero intrinsic process variability and long-term temporal variability (due to NBTI/PBTI) are considered for optimizing the FinFET device orientation combinations to improve the stability/variability of 6T FinFET SRAM cells with oxide and high- $k$ gate dielectrics, respectively. For NBTI/PBTI, the temporal degradation in SRAM stability/variability under worst case stress pattern/condition is considered. This paper is organized as follows. Section II describes the device design and simulation methodology used in this paper. Section III investigates the stability and variability of the 6T FinFET SRAM cells with various surface orientation combinations and gate dielectrics. In the first part of Section III, the fin LER is considered to optimize the 6T FinFET SRAM cells in terms of $\mu \mathrm{RSNM} / \sigma \mathrm{RSNM}$, where $\mu \mathrm{RSNM}$ is the mean of READ static noise margin (RSNM), and $\sigma \mathrm{RSNM}$ is the standard deviation of the RSNM. In the second part, the combined effects of fin 

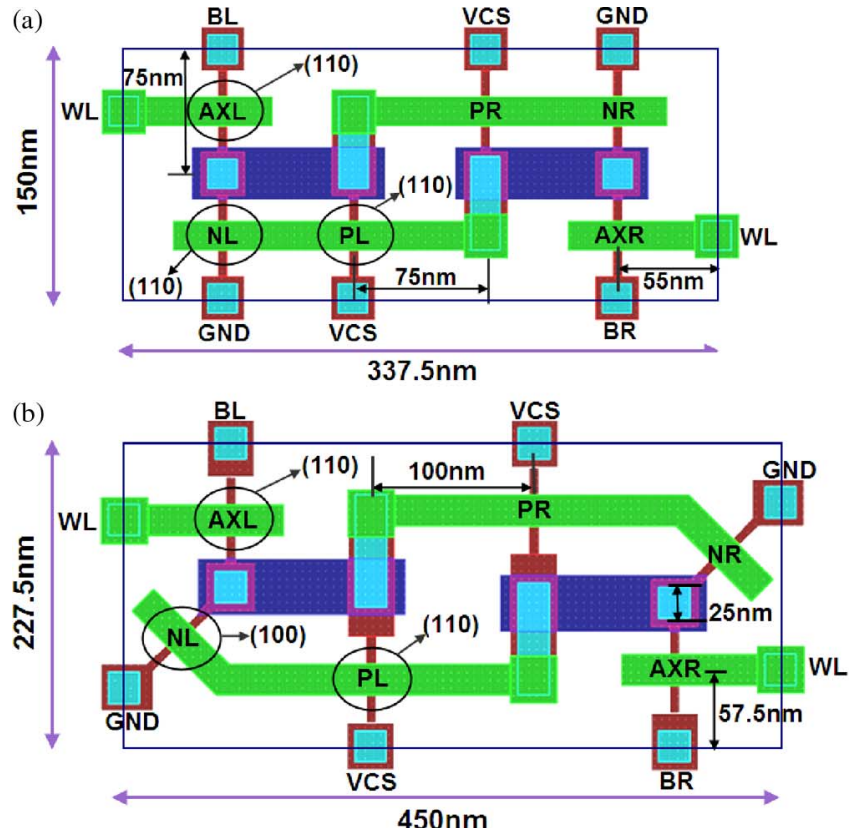

(c)

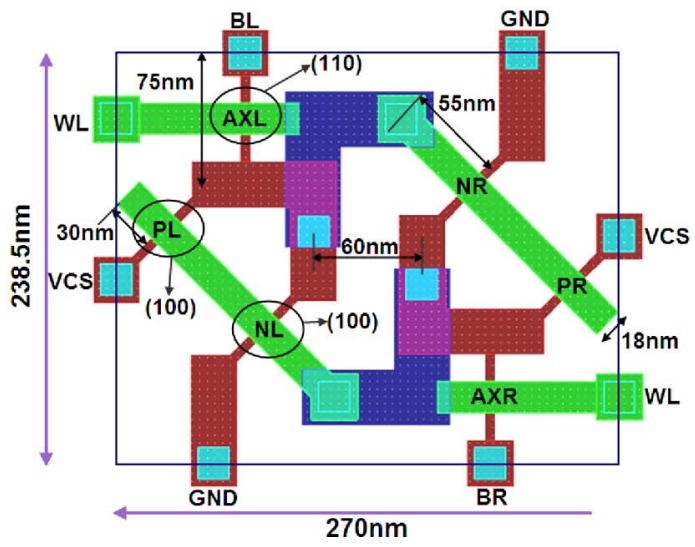

Fig. 1. (a) PU (PL/PR), PD (NL/NR), and PG transistors (AXL/AXR), all with (110) orientation. (b) (110) PU, (100) PD, and (110) PG transistors. (c) (100) PU, (100) PD, and (110) PG transistors.

LER and the NBTI/PBTI-induced temporal variability are then considered to optimize the 6T FinFET SRAM cells with oxide and high- $k$ dielectrics, respectively. Section IV concludes this paper.

\section{Device Design and Simulation Methodology}

In this paper, the 6T FinFET SRAM cells designed with $18-\mathrm{nm} L_{g}$ FinFET devices $\left[W_{\text {fin }}=5 \mathrm{~nm}, H_{\text {fin }}=15 \mathrm{~nm}\right.$, channel doping $=1 \mathrm{e} 17 \quad \mathrm{~cm}^{-3}, \quad V_{\mathrm{dd}}=1 \mathrm{~V}$, gate stacks: $\mathrm{SiO}_{2}(0.6 \mathrm{~nm}) / \mathrm{HfO}_{2}(2.5 \mathrm{~nm})$ or $\left.\mathrm{SiO}_{2}(1 \mathrm{~nm})\right]$ are analyzed using 3-D atomistic technology computer-aided design mixed-mode simulations [12]. The quantum-confinement effect is calibrated with the exact solution of Schrödinger's equation [13] to accurately account for the threshold voltage sensitivity to process variations for (100)/(110) N/PFETs. Reaction-diffusion model [14] is used to calibrate the threshold voltage drift due to NBTI/PBTI [10], [15]. To assess the dominant process variation source, i.e., fin LER [4], [16], the rough line edge patterns are generated using Fourier synthesis approach [17] with correlation length $=20 \mathrm{~nm}$ and

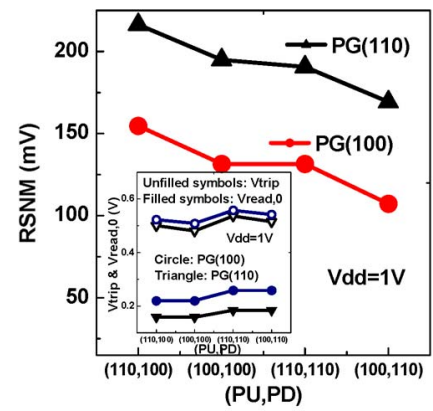

(a)

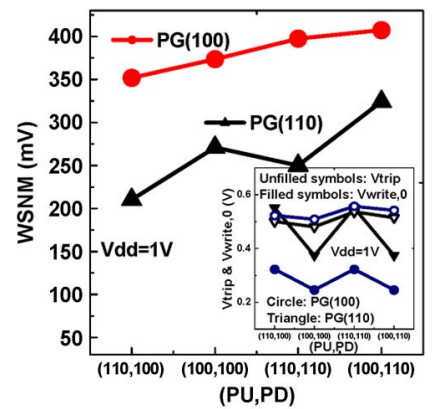

(b)
Fig. 2. (a) RSNM comparisons for eight types of 6T FinFET SRAM cells. (b) WSNM comparisons for eight types of 6T FinFET SRAM cells.

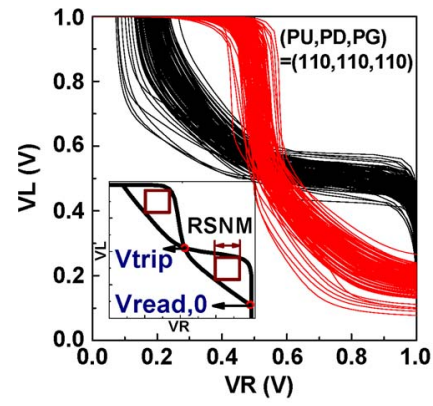

(a)

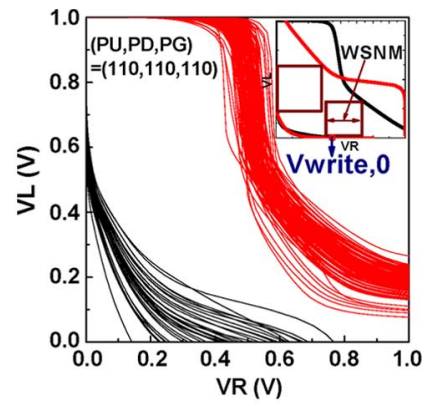

(b)
Fig. 3. (a) RSNM variation and (b) WSNM variation due to fin LER. (Correlation length $=20 \mathrm{~nm}$ and rms amplitude $=1.5 \mathrm{~nm}[4]$ ).

root mean square amplitude $=1.5 \mathrm{~nm}$ [4]. Atomic-level 3-D mixed-mode Monte Carlo simulations with 200 samples are then performed for each case.

\section{6T FINFET SRAM CELLS WITH (100)/(110) SURFACE ORIENTATIONS}

Pull-up (PU), pull-down (PD) and pass-gate (PG) transistors with (110) and (100) orientations can be combined for eight types of 6T FinFET SRAM cells. Fig. 2(a) shows the RSNM and Vread,0/Vtrip (defined in Fig. 3(a) inset) comparisons among the eight types of cells. The RSNM is defined as the minimum noise voltage present at each of the cell storage nodes necessary to flip the state of the cell. Vread,0 is the READ disturb voltage determined by the voltage divider effect between the PG and PD transistors. Vtrip is the voltage needed to flip the cell inverter. Increase in Vread,0 or decrease in Vtrip will degrade the RSNM. FinFET SRAM cells with (110) PG devices show lower Vread,0 and higher RSNM than that with (100) PG devices. Due to stronger (100) PD device, $(\mathrm{PU}, \mathrm{PD}, \mathrm{PG})=(110,100,110)$ and $(100,100,110)$ show lower Vread, 0 and higher RSNM than the standard SRAM cell with all (110) devices. Fig. 2(b) shows the WRITE static noise margin (WSNM) and Vwrite,0/Vtrip (defined in Fig. 3(b) inset) comparisons. The WSNM is determined by the smaller of the two squares that can fit between the cell-static voltage transfer characteristics during a WRITE operation (see Fig. 3(b) inset). Vwrite, 0 is determined by the voltage divider effect between the PU PFET and PG transistors. Lower Vwrite, 0 will benefit the WSNM. As can be seen, (100) PG device with stronger strength (higher mobility) shows lower Vwrite, 0 and larger WSNM. 


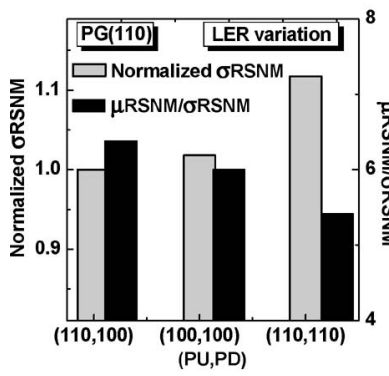

(a)

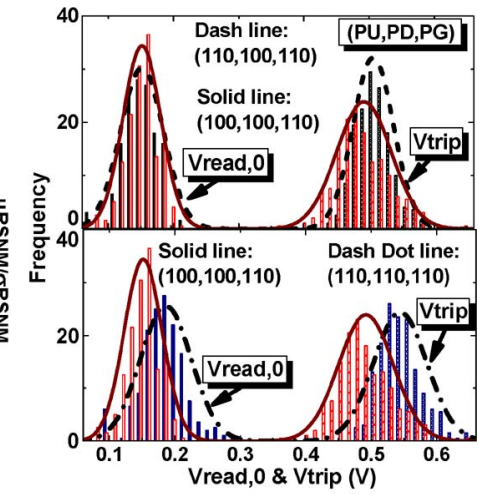

(b)
Fig. 4. (a) Normalized $\sigma$ RSNM and $\mu$ RSNM $/ \sigma$ RSNM comparison considering fin LER. $(110,100,110)$ SRAM cell shows largest $\mu$ RSNM $/ \sigma$ RSNM. (b) Vread,0 and Vtrip variation comparisons considering fin LER

\section{A. Time-Zero Stability/Variability Due to Process Variation}

In this section, the impacts of local random variations on device variability and optimization of the 6T FinFET SRAM cells are analyzed. Due to the difference in quantization effective mass [18]-[20], the effect of quantum confinement varies for different orientations. FinFETs with smaller quantization effective mass and stronger quantum confinement are more susceptible to fin LER than that with larger quantization effective mass. Fig. 3 illustrates the degradation READ/WRITE stability of 6T FinFET SRAM cell due to fin LER. Fig. 4(a) shows the normalized $\sigma$ RSNM and $\mu \mathrm{RSNM} / \sigma \mathrm{RSNM}$ comparisons among the three types of FinFET SRAM cells with higher RSNM. The SRAM cell with $(\mathrm{PU}, \mathrm{PD}, \mathrm{PG})=(100,100,110)$ shows larger $\sigma \mathrm{RSNM}$ than the $(110,100,110)$ case. Because (100) PU device with stronger quantum confinement exhibits larger threshold voltage variation due to fin LER than the (110) PU device, the $(100,100,110)$ SRAM cell shows larger Vtrip variation [see Fig. 4(b)] and $\sigma$ RSNM than the $(110,100,110)$ cell. The voltage margin between Vread,0 and Vtrip is larger in the $(110,100,110)$ cell than the $(100,100,110)$ one, which indicates that the $\mu \mathrm{RSNM}$ is larger in the $(110,100,110)$ SRAM cell. Therefore, the $(110,100,110)$ SRAM cell shows larger $\mu$ RSNM $/ \sigma$ RSNM than the $(100,100,110)$ one. The $(\mathrm{PU}, \mathrm{PD}, \mathrm{PG})=(110,110,110)$ SRAM cell shows higher $\sigma$ RSNM than the $(100,100,110)$ cell. The (110) NFET with stronger quantum confinement shows larger threshold voltage variation, due to fin LER, than the (100) NFET. Therefore, the $(110,110,110)$ SRAM cell with (110) PD device shows larger Vread,0 variation than the $(100,100,110)$ cell with (100) PD device [see bottom of Fig. 4(b)]. Vtrip is determined by the strength ratio between PU PFET and PD NFET devices. The $(110,110,110)$ cell with (110) PD NFET and the $(100,100,110)$ cell with (100) PU PFET show comparable Vtrip variation due to stronger quantum confinement in (110) PD NFET and (100) PU PFET, respectively, [see bottom of Fig. 4(b)]. Therefore, the $(110,110,110)$ cell with larger Vread, 0 variation and comparable Vtrip variation shows larger $\sigma$ RSNM than the $(100,100,110)$ cell.

Fig. 5(a) compares the normalized $\sigma \mathrm{WSNM}$ and $\mu \mathrm{WSNM} / \sigma \mathrm{WSNM}$. Vwrite, 0 is determined by the voltage divider effect between PU PFET and PG NFET devices.

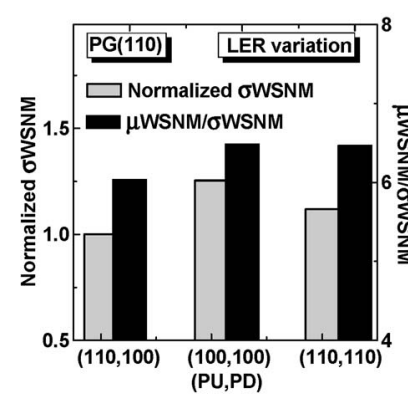

(a)

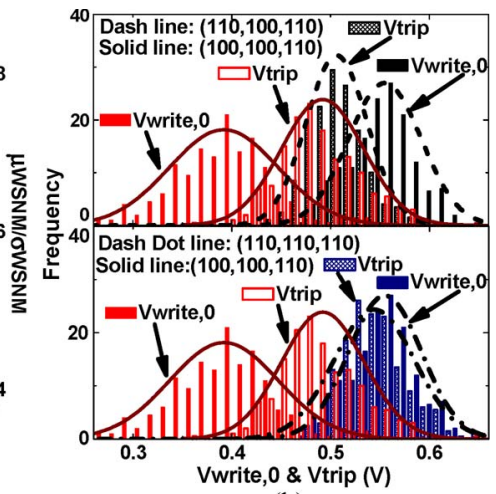

(b)
Fig. 5. (a) $(100,100,110)$ SRAM cell shows largest $\mu \mathrm{WSNM} / \sigma \mathrm{WSNM}$ (b) Vwritet,0 and Vtrip variation comparisons considering fin LER.

The $(100,100,110)$ cell with (100) PU PFET shows larger Vwrite, 0 variation and $\sigma$ WSNM than the $(110,100,110)$ and $(110,110,110)$ cells with (110) PU devices [see Fig. 5(b)]. Due to its larger voltage margin between Vtrip and Vwrite, 0 , the $(100,100,110)$ cell shows larger $\mu$ WSNM than the $(110,100,110)$ and $(110,110,110)$ cells. Even though the $(100,100,110)$ cell has larger $\sigma$ WSNM than the $(110,100,110)$ and $(110,110,110)$ cells, it still shows larger $\mu \mathrm{WSNM} / \sigma \mathrm{WSNM}$ due to its larger $\mu \mathrm{WSNM}$.

\section{B. Long-Term Stability/Variability Due to NBTI/PBTI}

Another factor of variability is the degradation of transistor parameters over time that also lowers the operating margin of SRAM cells. The NBTI (for PFET) and PBTI (for NFET) increase the transistor threshold voltages and reduce the drive currents with time. The NBTI/PBTI-induced random discrete charge trapping results in additional statistical variation. Fig. 6(a) and (b) show the time-dependent threshold voltage increase $\left(\left|\Delta V_{\text {th }}\right|\right)$ due to NBTI and PBTI for $\mathrm{SiO}_{2} / \mathrm{HfO}_{2} / \mathrm{TiN}$ and $\mathrm{SiO}_{2}$ FETs, respectively, and the insets demonstrate the good calibration results with published data [7], [8]. For $\mathrm{SiO}_{2} / \mathrm{HfO}_{2} /$ TiN FETs, PBTI- and NBTI-induced $V_{\text {th }}$ shifts are comparable. For $\mathrm{SiO}_{2}$ FETs, NBTI-induced $\left|\Delta V_{\text {th }}\right|$ is larger than PBTI by approximately one order of magnitude for the poly-gate FinFETs studied. The generated interface traps account for the increase in device threshold voltage as follows:

$$
\left|\Delta V_{\mathrm{th}}(t)\right|=q N_{\mathrm{it}}(t) / C_{g}
$$

where $N_{\text {it }}$ is the density of interfacial traps and $C_{g}$ is the gate capacitance. Based on this equation, the trap density for each case can be obtained (as shown in Table I). With the average number of traps determined for specific surface orientation, the actual number of traps in each device is randomly generated based on Poisson distribution [21]. Then, each trap is assigned to a random location in the channel/gate dielectric interface [23].

In this paper, the degradation in SRAM stability with time under worst case stress pattern/condition (extreme asymmetry condition, only PR with NBTI and NL with PBTI) is considered, as shown in Fig. 7(a). Fig. 7(b) shows that FinFET SRAM cells with $\mathrm{SiO}_{2}$ gate dielectric suffer from NBTI and show 9.5\% 


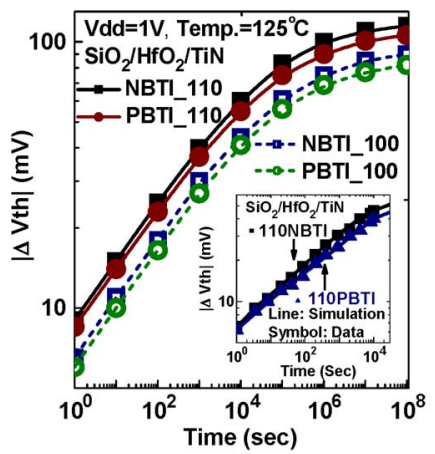

(a)

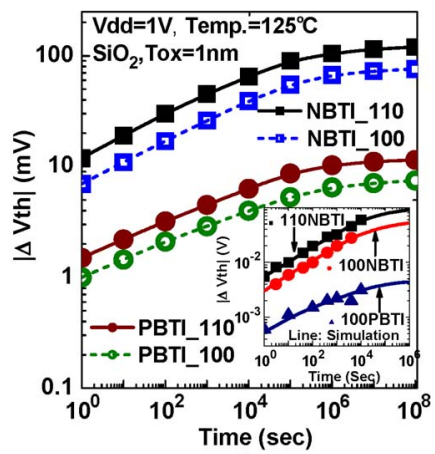

(b)
Fig. 6. (a) NBTI/PBTI-induced $V_{\text {th }}$ shift for $\mathrm{SiO}_{2} / \mathrm{HfO}_{2} / \mathrm{TiN}$ FET. (Inset) The model-data calibration. (b) BTI-induced $V_{\text {th }}$ shift for the $\mathrm{SiO}_{2}$ gate dielectric FET.

TABLE I

Trap Density For High- $k$ AND OXIDE GATE Dielectrics With (100) And (110) Surface Orientations. The Stress Time Is $1 \times 10^{8} \mathrm{~s}$, TEMPERATURE $=125^{\circ} \mathrm{C}$, AND Vdd $=1 \mathrm{~V}$

\begin{tabular}{|c|c|c|c|}
\hline Nit $\left(\mathrm{cm}^{-2}\right)$ & Surface Orientation & $\mathrm{HfO}_{2}$ & $\mathrm{SiO}_{2}$ \\
\hline \multirow{2}{*}{ NBTI } & $(110)$ & $2.48 \times 10^{12}$ & $2.58 \times 10^{12}$ \\
\cline { 2 - 4 } & $(100)$ & $1.94 \times 10^{12}$ & $1.52 \times 10^{12}$ \\
\hline \multirow{2}{*}{ PBTI } & $(110)$ & $2.32 \times 10^{12}$ & $2.48 \times 10^{11}$ \\
\cline { 2 - 4 } & $(100)$ & $1.65 \times 10^{12}$ & $1.62 \times 10^{11}$ \\
\hline
\end{tabular}

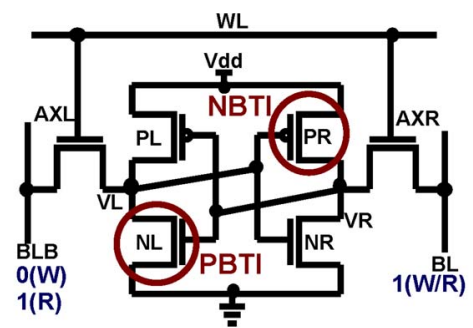

(a)

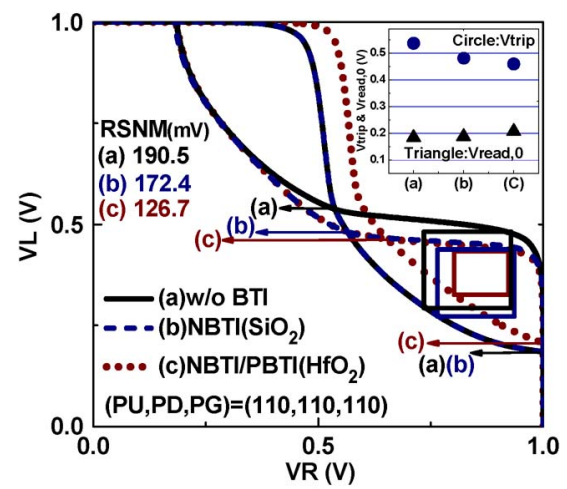

(b)

Fig. 7. (a) Worst case stress scenario for READ (R) and WRITE (W) stability. (b) RSNM comparison among curves (a) without BTI, (b) considering NBTI only, and (c) considering NBTI/PBTI. The stress time is $1 \times 10^{8} \mathrm{~s}$ at $125^{\circ} \mathrm{C}$.

degradation (stress time is $1 \times 10^{8} \mathrm{~s}$ at $125^{\circ} \mathrm{C}$ ) in RSNM due to its decreased Vtrip. FinFET SRAM cells with high- $k$ gate dielectric under the same stress time and temperature suffer from NBTI/PBTI and show 33.5\% degradation in RSNM due to its increased Vread, 0 and decreased Vtrip (see Fig. 7(b) inset). As shown, the sensitivity of PBTI on RSNM is larger than NBTI. Fig. 8 shows the impact of NBTI/PBTI-induced $\left|\Delta V_{\text {th }}\right|$ on the RSNM. The FinFET SRAM cells with (110)-oriented $\mathrm{PU}(\mathrm{PD})$ devices suffer larger NBTI(PBTI) degradation due to

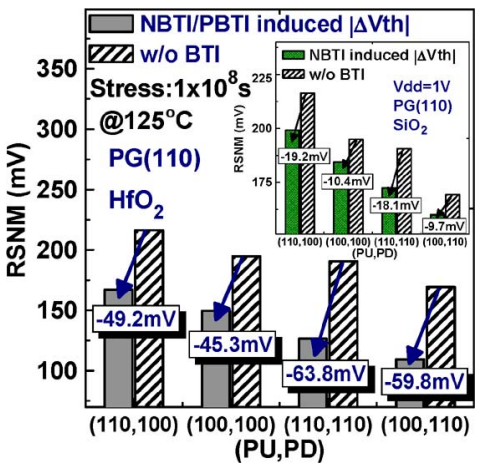

Fig. 8. RSNM degradation due to NBTI/PBTI. (Inset) The RSNM degradation due to NBTI only.

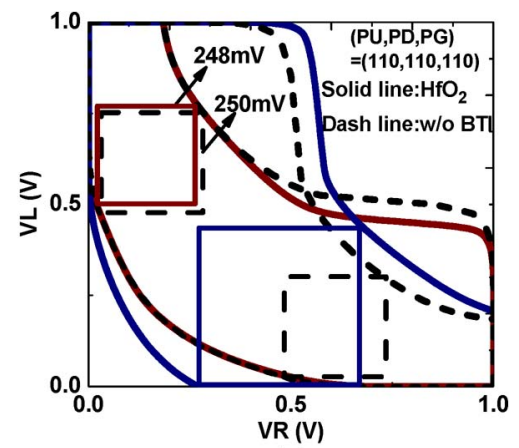

(a)

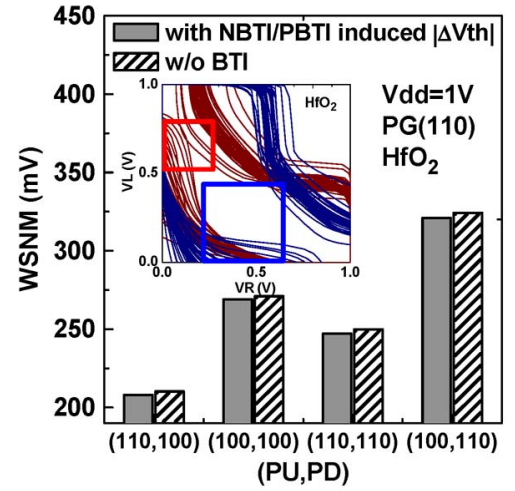

(b)

Fig. 9. (a) WSNM comparison between SRAM cells without BTI and considering NBTI/PBTI. The WRITE curves of solid and dashed lines overlap with each other. (b) Slight degradation in WSNM due to NBTI/PBTI under worst case stress condition. NBTI/PBTI stress time is $1 \times 10^{8} \mathrm{~s}$ at $125^{\circ} \mathrm{C}$.

higher number of interface traps, resulting in larger degradation in RSNM. In contrast with the significant RSNM degradation due to NBTI/PBTI, Fig. 9(a) and (b) show that the WSNM only slightly degrades. In Fig. 7(a), NBTI weakens PR and makes VR easier to write than VL; therefore, WSNM is mainly determined by writing VL. The long-term WSNM variability slightly degrades, as compared with the time-zero WSNM variability. Fig. 10 shows the long-term RSNM variability considering LER and NBTI/PBTI-induced $V_{\text {th }}$ variation for high- $k$ and oxide gate dielectric FETs. PBTI dominates the RSNM variation for high- $k$ metal gate SRAM cells; thus, SRAM cells with (110) PD devices show larger $\sigma$ RSNM, Vread, 0 variation [see bottom of Fig. 11(a)] and Vtrip variation [see bottom of Fig. 11(b)]. However, for $\mathrm{SiO}_{2}$ FETs, NBTI dominates its RSNM 


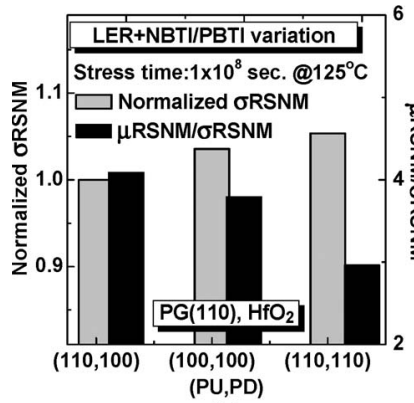

(a)

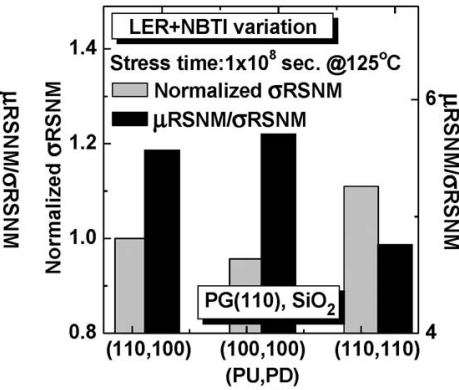

(b)
Fig. 10. (a) Normalized $\sigma$ RSNM and $\mu$ RSNM $/ \sigma$ RSNM comparison considering fin LER and NBTI/PBTI-induced variation. The $(110,100,110)$ SRAM cell $\left(\mathrm{HfO}_{2}\right)$ shows the largest $\mu$ RSNM $/ \sigma$ RSNM. (b) The $(100,100,110)$ SRAM cell $\left(\mathrm{SiO}_{2}\right)$ shows the largest $\mu \mathrm{RSNM} / \sigma \mathrm{RSNM}$.

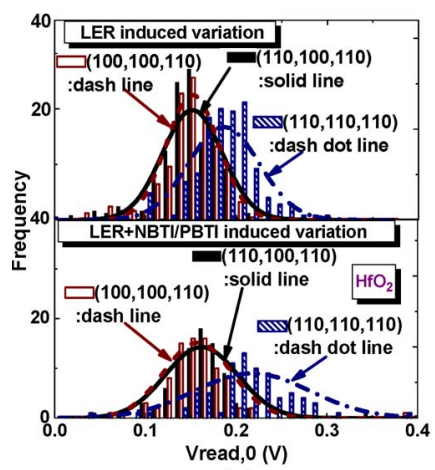

(a) (b)

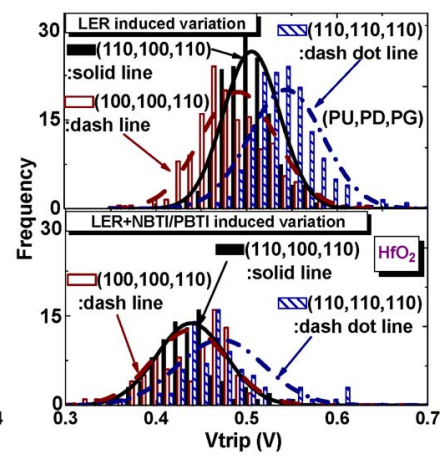

Fig. 11. (a) (110) PD devices show larger time-dependent Vread,0 variability degradation and Vread,0 increase. (b) (110) PD devices show larger timedependent Vread,0 variability degradation and Vread,0 increase. BTI stress time is $1 \times 10^{8} \mathrm{~s}$ at $125^{\circ} \mathrm{C}$.

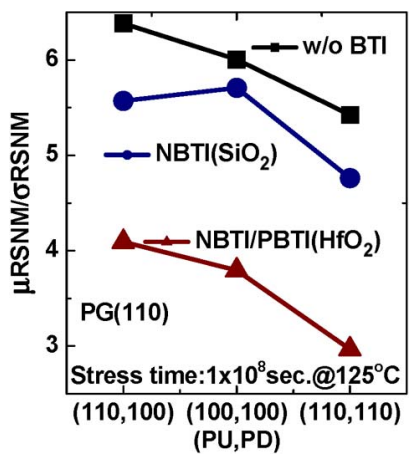

Fig. 12. $\mu$ RSNM $/ \sigma$ RSNM comparison considering short-term (fin LER) and long-term (fin LER + NBTI/PBTI) variations.

variation; thus, SRAM cells with (110) PU devices show larger decrease in $\mu$ RSNM (see Fig. 8 inset) and larger $\sigma$ RSNM [see Fig. 10(b)]. Therefore, $\mathrm{SRAM}$ cells $\left(\mathrm{SiO}_{2}\right.$ dielectric) with (110) PU devices show larger decrease in $\mu \mathrm{RSNM} / \sigma \mathrm{RSNM}$ than SRAM cells with (100) PU devices. Fig. 12 demonstrates that NBTI/PBTI-induced temporal variability in SRAM will change the optimal choice of FinFET SRAM cells with different gate stacks in terms of $\mu \mathrm{RSNM} / \sigma \mathrm{RSNM}$.

The fin LER-induced time-zero variability of FinFET SRAM cell is related to the fin width. FinFET SRAM cells with smaller fin width and larger quantum confinement will show larger difference in the time-zero variability among cells with

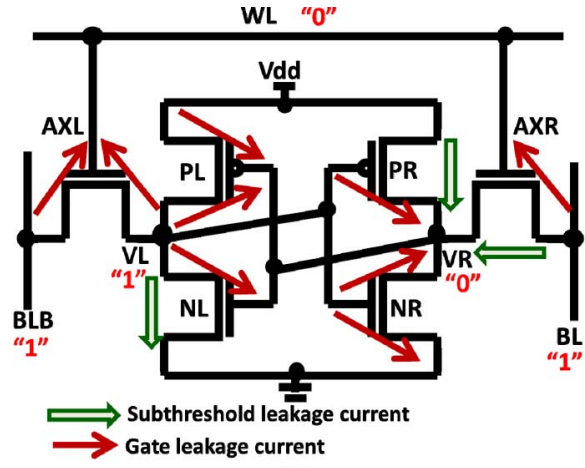

(a)

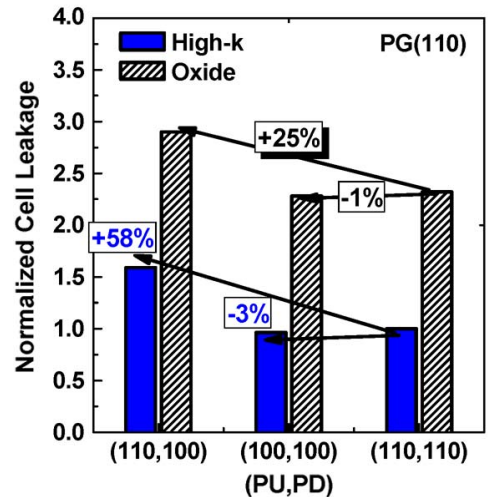

(b)

Fig. 13. (a) Cell leakage components of a 6T SRAM cell. (b) Normalized cell leakage comparisons of FinFET SRAM cells with high- $k$ and oxide gate dielectrics. The cell leakages of high- $k$ FinFET SRAM cells show larger orientation dependence than that of oxide FinFET SRAM cells.

different surface orientation combinations. On the other hand, FinFET SRAM cells with different surface orientations may show comparable time-zero variability if wider fin devices with less quantum confinement are used. However, the NBTI/PBTIinduced temporal variability still impacts the optimal choice of FinFET SRAM cells with different surface orientation combinations.

Fig. 13(a) shows the leakage components of the 6T SRAM cell. The standby leakage current of the 6T SRAM cell can be estimated by the sum of all the subthreshold and gate leakage currents. Fig. 13(b) shows the normalized cell leakage comparisons of FinFET SRAM cells with oxide and high- $k$ gate dielectrics. The subthreshold leakage current exponentially increases with decreasing threshold voltage. Therefore, the (110) NFET [(100) PFET] with stronger quantum confinement and larger threshold voltage shows lower subthreshold leakage current than the (100) NFET [(110) PFET]. Yang et al. [24] showed that the gate leakage currents are comparable between the (110) and (100) devices. In other words, the difference in cell leakages among these FinFET SRAM cells [see Fig. 13(b)] is mainly due to the difference in the subthreshold leakage currents. FinFET SRAM cells with high- $k$ gate dielectric show two orders of magnitude lower gate leakage than that with oxide gate dielectric. Therefore, the cell leakage currents of high- $k$ FinFET SRAM cells are mainly from the subthreshold leakage currents, thus exhibiting larger orientation dependence than that of oxide FinFET SRAMs. Compared with the $(110,110,110)$ cell, the $(110,100,110)$ cell with high- $k$ gate dielectric shows 
$58 \%$ higher cell leakage, whereas the $(110,100,110)$ cell with oxide gate dielectric shows $25 \%$ higher cell leakage.

\section{CONCLUSION}

We have investigated the impacts of fin LER and NBTI/PBTI on the stability and variability of the 6T FinFET SRAM cells with high- $k$ and oxide gate dielectrics, respectively. The 3-D mixed-mode simulations together with atomistic Monte Carlo simulations were used to investigate the variability due to fin LER and NBTI/PBTI-induced random discrete traps. The time-dependent $V_{\text {th }}$ drift and variation due to NBTI/PBTI degraded the stability and variability of RSNM (significantly) and WSNM (slightly). Our paper has indicated that the optimum FinFET SRAM design had to consider the combined effects of the intrinsic process variability, surface orientation, the specific gate dielectric used, and the temporal variability introduced by NBTI/PBTI

\section{ACKNOWLEDGMENT}

The authors are grateful to National Center for HighPerformance Computing in Taiwan for computational facilities and software.

\section{REFERENCES}

[1] K. Shin, C. O. Chui, and T.-J. King, "Dual stress capping layer enhancement study for hybrid orientation FinFET CMOS technology," in IEDM Tech. Dig., 2005, pp. 988-991.

[2] S. Gangwal, S. Mukhopadhyay, and K. Roy, "Optimization of surface orientation for high performance, low power and robust FinFET SRAM," in Proc. CICC, 2006, pp. 433-436.

[3] Z. Guo, S. Balasubramanian, R. Zlatanovici, T.-J. King, and B. Nikolic, "FinFET based SRAM design," in Proc. ISLPED, Aug. 2005, pp. 2-7.

[4] E. Baravelli, A. Dixit, R. Rooyackers, M. Jurczak, N. Speciale, and K. De Meyer, "Impact of line-edge roughness on FinFET matching performance," IEEE Trans. Electron Devices, vol. 54, no. 9, pp. 2466-2474, Sep. 2007.

[5] S. Mitra, "Circuit failure prediction for robust system design in scaled CMOS," in Proc. IRPSPhoenix, AZ, 2008, pp. 524-531.

[6] V. Huard, C. Parthasarathy, C. Guerin, T. Valentin, E. Pion, M. Mammasse, N. Planes, and L. Camus, "NBTI degradation: From transistors to SRAM arrays," in Proc. IRPS, Phoenix, AZ, 2008, pp. 289300.

[7] S. E. Rauch, III, "Review and reexamination of reliability effects related to NBTI-induced statistical variations," IEEE Trans. Device Mater. Rel., vol. 7, no. 4, pp. 524-530, Dec. 2007.

[8] K. Kang, H. Kufluoglu, K. Roy, and M. A. Alam, "Impact of negative-bias temperature instability in nanoscale SRAM array: Modeling and analysis," IEEE Trans. Comput.-Aided Design Integr. Circuits Syst., vol. 26, no. 10, pp. 1770-1781, Oct. 2007.

[9] A. Bansal, R. Rao, J.-J. Kim, S. Zafar, J. H. Stathis, and C.-T. Chuang, "Impacts of NBTI and PBTI on SRAM static/dynamic noise margins and cell failure probability," Microelectron. Reliab., vol. 49, no. 6, pp. 642649, Jun. 2009.

[10] S. Zafar, M. Yang, E. Gusev, A. Callegari, J. Stathis, T. Ning, R. Jammy, and M. Ieong, "A comparative study of NBTI as a function of Si substrate orientation and gate dielectrics ( $\mathrm{SiON}$ and $\left.\mathrm{SiON} / \mathrm{HfO}_{2}\right)$," in Proc. VLSITSA, 2005, pp. 128-129.

[11] S. J. Jang, D. H. Ka, C. G. Yu, K.-S. Kim, W.-J. Cho, and J. T. Park, "Comparative study of NBTI as a function of Si film orientation and thickness in SOI pMOSFETs," Microelectron. Reliab., vol. 47, no. 9-11, pp. 1411-1415, Sep.-Nov. 2007.

[12] Sentaurus TCAD, C2009-06 Manual.

[13] Atlas User's Manual, SILVACO, Santa Clara, CA, 2008.

[14] M. A. Alam and S. Mahapatra, "A comprehensive model of PMOS NBTI degradation," Microelectron. Reliab., vol. 45, no. 1, pp. 71-81, Jan. 2005.
[15] S. Zafar, Y. H. Kim, V. Narayanan, C. Cabral, Jr., V. Paruchuri, B. Doris, J. Stathis, A. Callegari, and M. Chudzik, "A comparative study of NBTI and PBTI (charge trapping) in $\mathrm{SiO}_{2} / \mathrm{HfO}_{2}$ stacks with FUSI, TiN, Re gates," in VLSI Symp. Tech. Dig., 2006, pp. 23-25.

[16] A. Dixit, K. G. Anil, E. Baravelli, P. Roussel, A. Mercha, C. Gustin, M. Bamal, E. Grossar, R. Rooyackers, E. Augendre, M. Jurczak, S. Biesemans, and K. De Meyer, "Impact of stochastic mismatch on measured SRAM performance of FinFETs with resist/spacer-defined fins: Role of line-edge-roughness," in IEDM Tech. Dig., 2006, pp. 1-4.

[17] A. Asenov, S. Kaya, and A. R. Brown, "Intrinsic parameter fluctuations in decananometer MOSFETs introduced by gate line edge roughness," IEEE Trans. Electron Devices, vol. 50, no. 5, pp. 1254-1260, May 2003.

[18] F. Stern and W. E. Howard, "Properties of Semiconductor surface inversion layers in the electric quantum limit," Phys. Rev., vol. 163, no. 3, pp. 816-835, Nov. 1967.

[19] A. Haque and M. Z. Kauser, "A comparison of wave-function penetration effects on gate capacitance in deep submicron n- and p-MOSFETs," IEEE Trans. Electron Devices, vol. 49, no. 9, pp. 1580-1587, Sep. 2002.

[20] M. Saitoh, S. Kobayashi, and K. Uchida, "Physical understanding of fundamental properties of Si (110) pMOSFETs-Inversion-layer capacitance, mobility universality, and uniaxial stress effects," in IEDM Tech. Dig., 2007, pp. 711-714.

[21] S. E. Rauch, "The statistics of NBTI-induced VT and $\beta$ mismatch shifts in pMOSFETs," IEEE Trans. Device Mater. Rel., vol. 2, no. 4, pp. 89-93, Dec. 2002.

[22] Y.-S. Wu, M.-L. Fan, and P. Su, "Impact of surface orientation on $V_{\text {th }}$ variability of FinFET," in Proc. Silicon Nanoelectron. Workshop, Honolulu, HI, Jun. 2010, pp. 1-2.

[23] M. F. Bukhori, S. Roy, and A. Asenov, "Simulation of statistical aspects of charge trapping and related degradation in bulk MOSFETs in the presence of random discrete dopants," IEEE Trans. Electron Devices, vol. 57, no. 4, pp. 795-803, Apr. 2010.

[24] M. Yang, V. Chan, K. Chan, L. Shi, D. Fried, J. Stathis, A. Chou, E. Gusev, J. Ott, L. Burns, M. Fischetti, and M. Ieong, "HybridOrientation Technology (HOT): Opportunities and challenges," IEEE Trans. Electron Devices, vol. 53, no. 5, pp. 965-978, May 2006.

[25] Y.-S. Wu and P. Su, "Impact of surface orientation on the sensitivity of FinFETs to process variations-An assessment based on analytical solution of Schrödinger equation," IEEE Trans. Electron Devices, vol. 57, no. 12, pp. 3312-3317, Dec. 2010.

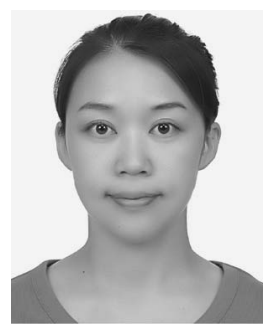

Vita Pi-Ho Hu (S'09) received the B.S. degree in the Department of Material Science and Engineering from the National Chiao Tung University, Hsinchu, Taiwan, in 2004, where she is currently working toward the Ph.D. degree in the Institute of Electronics.

Her research interests include characterization and modeling of ultrathin-body metal-oxidesemiconductor field-effect trasistors, analysis, and design of ultralow power static random access memories in nanoscaled technologies. 


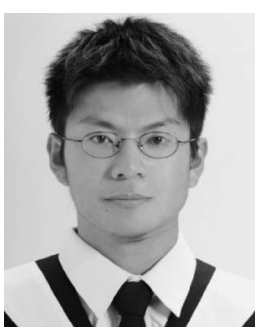

Ming-Long Fan (S'09) was born in Taichung, Taiwan, in 1983. He received the B.S. and M.S. degrees from National Chiao Tung University, Hsinchu, Taiwan, in 2006 and 2008, respectively, where he is currently working toward the Ph.D. degree in the Institute of Electronics.

His current research interests include design and modeling of subthreshold static random access memory in scaled/exploratory technologies.

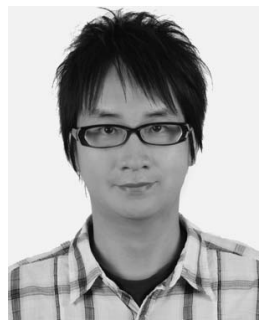

Chien-Yu Hsieh was born in Hsinchu, Taiwan, in 1985. He received the B.S. degree from the Department of Electrical Engineering National Chung Hsing University, Taichung, Taiwan, in 2008, and the M.S. degree from the Department of Electronics Engineering and Institute of Electronics, National Chiao Tung University, Hsinchu, Taiwan, in 2010.

His current research interests include design of static random access memory in scaled/exploratory technologies.

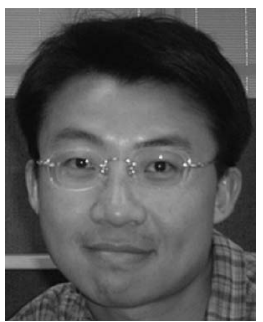

Pin Su (S'98-M'02) received the B.S. and M.S. degrees in electronics engineering from National Chiao Tung University, Hsinchu, Taiwan, and the Ph.D. degree from the University of California, Berkeley.

From 1997 to 2003, he conducted his doctoral and postdoctoral research in silicon-on-insulator (SOI) devices at Berkeley. He was also one of the major contributors to the unified BSIMSOI model, which is the first industrial standard SOI metal-oxide-semiconductor (MOS) field-effect transistor model for circuit design. Since August 2003, he has been with the Department of Electronics Engineering, National Chiao Tung University, where he is currently an Associate Professor. He has authored or coauthored over 100 research papers in refereed journals and international conference proceedings. His research interests include silicon-based nanoelectronics, modeling and design for exploratory complementary MOS (CMOS) devices, and device/circuit interaction and cooptimization in nano-CMOS.

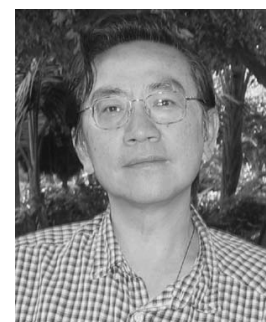

Ching-Te Chuang (S'78-M'82-SM'91-F'94) received the B.S.E.E. degree from National Taiwan University, Taipei, Taiwan, in 1975 and the Ph.D. degree in electrical engineering from the University of California, Berkeley, in 1982.

From 1977 to 1982, he was a Research Assistant with the Electronics Research Laboratory, University of California, working on bulk and surface acoustic wave devices. He joined the IBM T. J. Watson Research Center, Yorktown Heights, NY, in 1982. From 1982 to 1986, he worked on scaled bipolar devices, technology, and circuits. He studied the scaling properties of epitaxial Schottky barrier diodes, did pioneering works on the perimeter effects of advanced double-poly self-aligned bipolar transistors, and designed the first subnanosecond 5-Kb bipolar emitter-coupled logic static random access memory (SRAM). From 1986 to 1988, he was the Manager of the Bipolar Very Large Scale Integration (VLSI) Design Group, working on low-power bipolar circuits, high-speed high-density bipolar SRAMs, multi-Gb/s fiberoptic data-link circuits, and scaling issues for bipolar/bipolar complementary metal-oxide-semiconductor (CMOS) devices and circuits. Since 1988, he has managed the High Performance Circuit Group, investigating high-performance logic and memory circuits. Since 1993, his group has been primarily responsible for the circuit design of IBM's high-performance CMOS microprocessors for enterprise servers, PowerPC workstations, and game/media processors. Since 1996, he has been leading the efforts in evaluating and exploring scaled/emerging technologies, such as partially depleted silicon-on-insulator (SOI), ultrathin body/SOI, strained-Si devices, hybrid orientation technology, and multigate/fin-shaped field-effect transistor devices, for high-performance logic, and SRAM applications. Since 1998, he has been responsible for the Research VLSI Technology Circuit Codesign strategy and execution. His group has also been very active and visible in leakage/variation/degradation tolerant circuit and SRAM design techniques. He took early retirement from IBM to join National Chiao Tung University, Hsinchu, Taiwan, as a Chair Professor with the Department of Electronics Engineering in February 2008. He is the holder of 31 U.S. patents, with another 14 that are still pending. He has authored or coauthored over 290 papers.

Dr. Chuang has received one Outstanding Technical Achievement Award, one Research Division Outstanding Contribution Award, five Research Division Awards, and twelve Invention Achievement Awards from IBM. He was the corecipient of the Best Paper Award at the 2000 IEEE International SOI Conference. He has received the Outstanding Scholar Award from Taiwan's Foundation for the Advancement of Outstanding Scholarship for 2008 to 2013 He served on the Device Technology Program Committee for International Electron Devices Meeting in 1986 and 1987 and the Program Committee for Symposium on VLSI Circuits from 1992 to 2006. He was the Publication/ Publicity Chairman for Symposium on VLSI Technology and Symposium on VLSI Circuits in 1993 and 1994, and the Best Student Paper Award Subcommittee Chairman for Symposium on VLSI Circuits from 2004 to 2006. He was elected an IEEE Fellow in 1994 "For contributions to highperformance bipolar devices, circuits, and technology." He has authored many invited papers in international journals such as International Journal of High Speed Electronics, Proceedings of IEEE, IEEE Circuits and Devices Magazine, and Microelectronics Journal. He has presented numerous plenary, invited, or tutorial papers/talks at international conferences such as the International SOI Conference; Design Automation Conference; VLSI-Technology, Systems and Application; International Solid State Circuits Conference Microprocessor Design Workshop; VLSI Circuit Symposium Short Course; International Symposium in Quality Electronic Design; International Conference on ComputerAided Design; Asia-Pacific Microwave Conference; VLSI-Design, Automation and Test; International Symposium on Circuits and Systems; Memory Technology, Design, and Testing; World Scientific and Engineering Academy and Society; VLSI Design/Computer-Aided Design Symposium; and International Variability Characterization Workshop. 